\title{
Mapping Microbiome Signatures Associated With Antibiotic Usage On A Swine Production Farm Using Amplicon And Shotgun Data
}

Adrian Muwonge ( $\nabla$ adrian.muwonge@roslin.ed.ac.uk)

The University of Edinburgh The Roslin Institute https://orcid.org/0000-0002-8579-0384

Jolinda Pollock

SRUC Edinburgh Campus: Scotland's Rural College

Shih Barbara

The University of Edinburgh The Roslin Institute

Michael R. Hutchings

SRUC Edinburgh Campus: Scotland's Rural College

Mark Bronsvoort

The University of Edinburgh The Roslin Institute

Geoffrey Mainda

Zambia MFL: Zambia Ministry of Livestock and Fisheries Development

David L. Gally

The University of Edinburgh The Roslin Institute

Alexander Corbishley

The University of Edinburgh The Roslin Institute

\section{Research Article}

Keywords: Microbiome, Antibiotic, Resistance, Pig, Production, United Kingdom

Posted Date: June 29th, 2021

DOI: https://doi.org/10.21203/rs.3.rs-644289/v1

License: (c) (i) This work is licensed under a Creative Commons Attribution 4.0 International License. Read Full License 


\section{Abstract}

Background: High antimicrobial usage in swine production has the potential to create reservoirs of antimicrobial resistance (AMR) genes which are transferable to human pathogens via mobile genetic elements. Understanding microbial community responses to antibiotic use is central to unravelling transfer of such resistance genes. Our previous investigation revealed a scenario of optimal antibiotic activity associated with saturation of AMR genes on this farm. Here, we use amplicon and shotgun sequence data to investigate the microbiome signatures that underwrite such a phenomenon.

Results: We generated 1.24 and 576 million high quality 16S rRNA gene amplicon and shotgun sequences from 24 porcine faecal samples, respectively. The ratio of taxa detection at genus level between the two methods was 1:24. Using shotgun sequence data, 235 unique AMR genes, 122 modes of action and 17 antibiotic classes were identified using the MEGARes AMR database. Antibiotic usage in growing pigs was significantly associated with microbial and AMR resistome structural and compositional changes detectable two weeks after antibiotic initiation. These were characterised by a down regulation of MDR efflux pumps and an up regulation of macrolide-specific efflux pumps in the growing pigs (treated-group) linked to lower abundance of Verrucomicrobiaeceae. In the sows (non-treated group), a potentially undetected infection, was characterised by a high abundance of pathogenic viral sequences, microbial structural changes i.e. family Alcaligenaceae, and an up regulation of beta-lactamases, including MDR efflux pumps. We assembled 682 near complete bacterial genomes revealing that a large proportion of the resistome is carried by Firmicutes and Proteobacteria, specifically multi-class gene carriage by Clostridium species and Escherichia coli, which occurred exclusively in the treatment group.

Conclusion: Microbiome signatures i.e. microbial structure, composition and resistome carriage associated with antibiotic-use can be cost effectively screened with amplicon sequencing but their granularity unravelled using shotgun metagenomic data.

\section{Background}

Antibiotic usage is integral to livestock production globally [1, 2]. Until 2006, drugs such as tylosin and tetracycline were used to improve feed conversion efficiency in swine and poultry production systems in Europe [3]. Today, their use is restricted to therapeutic and where justifiable, prophylactic use, with the aims of limiting welfare and production impacts of infectious diseases [4]. However, global projections indicate that twothirds of the growth in antimicrobial usage is expected to be within the veterinary sector, with a doubling in use for pigs [5]. This raises concerns about the role pig farms can play as reservoirs of antimicrobial resistance (AMR) genes that could be transferred to human and pig pathogens via mobile genetic elements [6]. Unravelling the microbial characteristics that underwrite these genetic transfer mechanisms is important to inform the development of control strategies [7], however, recent evidence suggest that the vast majority of unexplored AMR is harboured by non-pathogenic and non-culturable bacteria present in human and animal gut microbiomes [8, 9]. Advances in sequencing technologies [8, 10] and improved efficiency in data processing have provided access and ability to examine this non-culturable fraction in epidemiologically relevant settings. However, there are few longitudinal studies that have interrogated AMR gene generation and dissemination in livestock production systems to date. Such approaches are essential to disentangle the contribution of temporal and contextual microbiome variation in high antibiotic use systems such as swine production [11]. Our previous work [11] indicated a state of saturation of AMR genes in a swine production system, with no apparent impact on the utility of antibiotics to limit infections and mortality. Here, we seek to unravel the microbiome characteristics and the association with antimicrobial resistance genes, referred to as the resistome, in the pig faecal samples on this farm. To do so, the resolution of shotgun sequence data was exploited and this allowed comparison with 16S rRNA gene sequence data from the same samples. We targeted four critical time points as part of the pig production cycle, i.e. at farrowing, weaning, growing and finishing where batch antibiotic treatment (metaphylaxis) is considered necessary to limit the impact of infectious diseases. It is noteworthy that welfare concerns precluded the use of within group controls, therefore sows on the same farm were sampled at the same timepoints as the production animals as a comparative control for changes which were also analysed over time within groups.

\section{Materials And Methods}

\section{Farm description}

This experiment was approved by the Royal (Dick) School of Veterinary Studies Veterinary Ethics Research Committee. A 600 sow (Landrace $x$ Large White) commercial farrowing to finishing unit in the United Kingdom was recruited for this study. On this unit, piglets (antibiotic treated group) were batch farrowed every four weeks and the sample collection commenced one week before the October 2016 batch farrowing process. Dry sows (Nontreated group) were housed in straw yards (referred to as the "sow barn") and nursing sows and piglets were housed on slats. Detailed herd and antimicrobial usage records have been published in our previous work [11].

\section{Study design and sampling}

The main study design and sampling procedures were reported in our previous publication [11]. Here, we focus on a subset of faecal samples collected at four time points - 9th November 2016, 30th November 2016, 14th December 2016 and 5th April 2017 - labelled as T1, T2, T3 and T4, respectively. These time points were selected on the basis of differing growth stages and antimicrobial treatment phases during the production cycle of the growing pigs. In parallel, faecal samples from the dry sow barn (where routine antimicrobial administration did not occur) were taken at each of 
these time points $(n=3)$ as shown in Fig. 1 a. Faecal samples were collected in spooned universal tubes prior to being stored immediately at $-20^{\circ} \mathrm{C}$ onsite. Samples were batch transported to the laboratory on dry ice for DNA extraction and dry matter calculations, as described previously [11]. Here we refer to this analysis as the selected amplicon resistome.

\section{Sequencing}

The extracted DNA was submitted to Edinburgh Genomics (United Kingdom) for shotgun metagenomic sequencing. Briefly, Illumina TruSeq DNA libraries were prepared from the DNA extracts on the HiSeq 4000 platform to generate 150bp paired end reads (Illumina, United States). The raw sequences for this study are publicly available on EMBL-EBI under accession number PRJEB34736. Reads generated from host DNA and Phix (Phix174) were filtered out by mapping the reads to the Sus Scrofa reference genome GCA_000003025 version 11.1 and Phix174 respectively using the run_contaminant_filter.pl script in Microbiome Helper suite [12]. Adapters and low-quality reads were then trimmed and removed respectively using Trimmomatic[13], the filtered reads were then taken forward for analysis. Detailed description of the 16S rRNA gene sequencing is reported here [11] but in brief; the paired end reads from the sequencing step above were processed using the Quantitative Insights Into Microbial Ecology-2 ${ }^{\text {TM }}$ (QIIME-2) software. The reads were then de-replicated and chimeric sequences removed before the denoising was done in DADA2 with output here as a feature table used for the downstream analysis. OTU taxonomic classification was done using a naïve Bayes classifier trained on the SILVA database (version March 2020) at 97\% similarity. Visualisation of taxonomic abundances and alpha diversity was done using ggplot[14] and plotly in R.

We also track the copy number of four selected AMR genes i.e. tetQ, tetB, ermB and dfra1 as using qPCR across time as earlier reported [11].

\section{Metagenomic assembly}

Metagenomic assemblies henceforth referred to as contigs were generated from each of the samples using Megahit (v1.1.3) with following parameters; -kmin-1pass, -m 2.5e +11 - presets meta-sensitive -min-contig-len 500 options -num_threads 16 [15]. The resultant contiguous sequences (contigs) contained unique chromosomal identifiers ( $\mathrm{UCl})$ which were used to track them through our database mapping process (Fig. 1a).

\section{Mapping contigs to AMR, plasmid and taxonomic databases}

In order to maximize the amount of sequences used, rather than annotating full genomes, we mapped the Megahit-assembled contigs to databases and then filter output using stringent thresholds. The aim here is to detect contigs carrying AMR genes (full resistome), plasmid and identifiable taxonomic sequences. Whilst this approach would not provide details of taxa carrying genes lower than Phylum, it allows us to see a more comprehensive picture for the composition of these genetic elements on a sample-wide basis. To identify contig carrying AMR genes ,we used Abricate[16] to map the contigs against the MEGARes[17] - https://megares.meglab.org. Here the output was filtered by contig length ( $\mathrm{n}>=800 \mathrm{bp}$ ) and percentage identity ( $>=75 \%)$.

Contig taxonomic assignment-Further taxonomic identification of contigs was performed using the CAT and BAT pipeline[18] which identifies assembled sequences up to genus level. Here, contigs were filtered according to length and retained taxonomic assignment based on at least three known markers [18]. This taxonomic assignment was validated using the Plasflow pipeline [19] which also predicts plasmid sequences and taxonomy at phylum level.

Merging output for analysis- The AMR gene and taxonomic mapping and assignment process generated a tab separated value (tsv) file with eight standard BLAST output columns for the former http://www.metagenomics.wiki/tools/blast/blastn-output-format-6

Using the UCl, AMR gene and taxonomic assignment data was merged into one file for downstream data analysis.

Analysis and Visualization- To visualise the entire resistome we use geom-tile in ggplot[14] while the taxonomic hierarchical clustering of 16S rRNA gene and shotgun data was done using the complexheatmaps [20] package in R. To detect differential abundance in the resistome and microbial populations, the negative binomial additive model in Deseq2 was applied [21]. Note that analysis of taxonomic abundance was done for bacterial, viral and eukaryotic sequences by filtering at kingdom level.

\section{AMR gene content inference}

We developed a mixed effects Poisson regression models [22] implemented using the Ime4 package [23] in R to analyse within and between groups variation in abundance of AMR genes at the four time points. To do so we make the following assumptions as showing in (Fig. 1b); a) The piglet's microbiome gut will exhibit more variation over time than the sows, b) sows will have a higher absolute count/abundance and richness of microbiome units (taxa or genes), c) Therefore, the gut microbiome changes $(\Delta \mathrm{m}=\Delta \mathrm{x}+\Delta \mathrm{y})$ will be a function of the change in temporal signal ( $\Delta \mathrm{x})$, developmental changes and routine activities such as antibiotic use $(\Delta y)$. We then defined the outcome as the read-normalised gene abundance. To compare between groups, we created subsets of the data i.e. Piglets and Sows (Farrowing + Dry), then developed a mixed effects Poisson model for each data set, with the fixed component as antibiotic class (AC) and time (T) point while the random component using the sample identification (SI). On its own, the random component represents within sample variation, i.e. the total variance in gene count for a sample including developmental effects, antibiotic use and seasonal variability) (Fig. 1b). The difference between the total variance and that which is explained by the fixed 
component is the unexplained variance. Comparing this between groups allows us to partition the variance and account for the antibiotic usage. For example;

$$
\text { unexplained variance }=\frac{\text { Total variance }- \text { Total explained variance }}{\text { Total variance }}
$$

Here we assume that most of the unexplained variance is likely due to antibiotic use. To compare the outputs from the models from the subsets above, we use jtools[24]. To assess the validity of the model, we assess if the data supports our assumptions. In addition, we use the modes of action[17] as the label in the same model to infer the functional characteristics of the gene abundance estimates. Model interpretation; a) Statistically significant variables in fixed component of the model for a dataset represented explanatory factors for within group variations, $b$ ) variables that were statistically significant between models (between datasets) represent factors with an overlapping effect between groups and c) distinct lack of overlap of variable estimates between group (between datasets) represents factors with a differential affect at group level.

\section{AMR gene carrying taxa}

To investigate the taxonomic carriage of AMR genes in this system, first near complete genomes were assembled 'de novo' from the metagenomic sequences using MEGAHIT (v1.1.3) with the options '-presets meta-sensitive -min-contig-len 500 -kmin-1pass'[15]. The resultant contigs were used as reference for BWA MEM[25, 26] read alignment with 4 threads, followed by metagenome binning with MetaBAT[1] (v0.32.4) using the parameters 'verysensitive --minContig 2000 -t 4'. The metagenome bins were then used as the input for MAGpy[27]; each bin represented a genome for an individual species. MAGpy also implemented genes-finding and translation thorough Prodigal (v2.6.3) [28], this included AMR genes as well as plasmid[29] The genome completeness was assessed using CheckM (v1.0.7) [30] and all genomes with completeness $>75 \%$ were collated and used to generate a phylogenetic trees with PhyloPhIAn (v3.0) [31]. Taxonomy classification was carried out by searching the Prodigal results against Uniport TrEMBL[32] database (version on 11th December 2020) using Diamond[33]. For each MAG, the presence of AMR genes and plasmids were assessed through Abricate[16] using the MEGARes [17]and plasmidfinder databases[16, 29] .

\section{Results}

\section{Sample sequence characteristics on amplicon and shotgun}

16S rRNA gene sequencing of 24 faecal samples generated 1.55 million 150bp paired-end reads, 95\% of these yielding at least Q30 values per sample. Assembly and filtering by sequencing depth (4000 sequences) retained 1.24 million sequences, which clustered into 1284 operational taxonomic units, 12 phyla and 141 genera. The mean alpha diversity based on the Shannon index was 2.2 across all the samples, 2.6(SD = 1.8-3.1) for piglets, $2.3(S D=1.28-3.0)$ for dry sows and $1.8(S D=1.3-1.8)$ for farrowing sows. With the exception of T3, alpha diversity was stable among sows but a significantly higher diversity for piglets at T3 and T4 (Fig S1).

Shotgun sequencing generated 456 gigabytes of data, i.e. 27.8 million sequences of $150 \mathrm{bp}$ paired end reads per sample. After quality control, each sample on average retained 25 million sequences, which were assembled into 9.2 million contigs. $47 \%$ of these contigs were $\geq 800 \mathrm{bp}$ of which only 9434 mapped to the MEGARes AMR database with a percentage identity $\geq 75 \%$. On the other hand, 650,368 unique contigs of $\geq 800 \mathrm{bp}$ were taxonomically classified to at least genus level.

\section{Taxonomic profile}

In general, the taxonomic profiles were comparable between the two methods but the difference in granularity was evident at family level (Fig $2 \mathrm{~A} \& \mathrm{C}$ ), i.e. The ratio of taxa detection at genus level between the two methods was 1:24, none the less, there were some discernible differences between pig groups across time (Fig 2B). Using 16S rRNA at phylum level, Firmicutes, Bacteriodetes and Proteobacteria constituted nearly $94 \%$ of gut microbiota with a marked increase in Bacteriodetes for dry sows at T3. The granularity of these is revealed by shotgun sequence data (Fig 2D), however, here the change in Bacteriodetes abundance was less dramatic and showed that same phylum was differentially higher among piglets at T3. Moreover, Shotgun sequence data revealed distinct clustering by time and group, for example; cluster 4 (Fig 2 A \& C) represented samples with the highest richness and these exclusively belonged to dry sows at T3. Cluster 3 (Fig $2 \mathrm{C}$ ) exclusively belonged to piglets at T4, therefore, richness peaks at different time points in piglets and sows. This observation supports our model assumptions. Cluster 1 exclusively belonged to piglets distinctly separated into T3 and T2 while cluster 2 mostly belonged to sows, this too reveals distinct clusters by time. Overall, this clustering indicates a strongtaxonomic signal associated with group and time.

At family level, Shotgun data shows that Bacteriodaceae, Verrumicrobiaceae and Bradymonadaceae were differentially abundant between piglets and sows (Fig S2a), with the Verrumicrobiaceae and Bradymonadaceae more abundant among sows (Fig S2b). Analysis of viral sequences revealed a steady increase in viral sequences in piglets over time, while that of sows was generally stable (Fig S3a and b). This was with exception of the dry sows at T3 which exhibited a marked increase in viral sequences which mapped to Siphoviridae, Phycodnaviridae and Podoviridae. Similarly, the hierarchical clustering of these viral sequences also distinctly separates dry sows at T3, however these characteristics were not mirrored by the Eukaryotic sequence data (Fig S3c).

\section{The antibiotic resistome}


Selected amplicon resistome presented as AMR gene copy number normalised by 16S rRNA gene copy number[11] is shown in Fig. 3A. At farrowing (T1), the sows carried significantly more tetQ and ermB gene copies with the former exhibiting the least variability in gene copy (P<0.001). This trend was mirrored by dry sows at the same time point. Among piglets, the abundance of tet $Q$ and erm $B$ genes was generally higher than sows across the three time points but the highest level was observed at T4 $(P<0.01)$, specifically, the abundance of these two genes was 2 -fold higher than that for dry sows. T3 was characterised by limited variability across all the five genes but the copy number of tetQ and erm $B$ remained markedly higher among piglets however, this was not the case for $d f r a 1$. Analysis of the same genes using shotgun sequence data Fig. 3B showed similar levels of tet $Q$ across time and groups. The trend of tetQ is similar to that observed with amplicon sequence data, especially at T3 and T4.

Full shotgun sequence resistome is shown in Fig. $3 \mathrm{C}$ and is comprised of 235 unique genes clustering into 17 antibiotic classes with 122 corresponding modes of action. In piglets, the abundance of genes encoding resistance for tetracycline generally increased over time; specific examples include tetW, tetQ, tetO, tet44 and tet32. By contrast, all of these genes had exhibited the opposite trend among sows (Fig S4a). This too supports our model assumptions. Macrolides, lincosamides and streptogramines (MLS) resistance-encoding genes exhibited comparatively low variation and all but Inuc increased over time (Fig S4a). In general, T4 was characterised by an increase in $m p h B, m a c B$ and erm $T$ - macrolide resistance genes - in piglets. Beyond the administered antibiotics, fluoroquinolone and glycopeptide-associated resistance genes also exhibited a gradual increase in abundance over time. For example; among sows, the abundance of genes that encode resistance for fluoroquinolones (parc gyrA and gyrB), beta-lactams (imp and carB), aminoglycosides (ant3-prime) and CCR (sme,mexW,mexK \& mexB) differentially increased at T3 (Fig S4b)

\section{AMR gene count inferences}

Almost all attributable variance at group level belonged to piglets, and after accounting for the temporal effect, approximately $66 \%$ of the variance remained explained, which is likely the effect size due to antibiotic usage in this group. There was a significant difference in within and between groups at T4 (Fig. 4A and Fig S5). The changes in gene count at T3 were statistically significant within the dry sows.

At antibiotic class level, salient differences in genes within-groups were detectable for all but cationic antimicrobial peptides, metronidazole in piglets and tetracyclines in sows. It is noteworthy that the difference in variance between sows and piglets also validates our model assumptions (Fig. 1 \& Fig. 4b). In addition, Fig S3b suggests a gradual increase in abundance and diversity of viral sequences in piglets over time. Finally, although the resistome (Fig. 3C) shows an increase in diversity of glycopeptide resistance genes, it did not translate into a significant differential abundance between piglets and sows.

When we consider the mode of action for resistance rather than the class of antibiotic (Fig. 4C), the results also indicate differential abundance within groups at T3 and T4, but a clear difference between groups at T3. Generally, the abundance here was skewed toward sows, but multi drug efflux pumps, polymyxin B resistance regulator and class B beta-lactamases were markedly higher in sows (Fig S6). Among piglets, the abundance of dihydrofolate reductase, vancomycin $\mathrm{G}$ type resistance protein, penicillin binding protein, MDR regulator and aminocoumarin- resistant DNA topoisomerases significantly varied (Fig. 4C \& S6). On the other hand, EF-Tu inhibition, fluoroquinolone DNA resistant topoisomerases, and ATPbinding cassettes $A B C$ efflux pumps differentially varied within sows over time. Finally, mechanisms such as resistant $30 S$ ribosomal subunit protein S12, class A beta-lactamases, macrolide resistance efflux pumps and tetracycline ribosomal protection protein were differentially abundant within both groups.

\section{Antibiotic resistance gene carriage by taxa}

Using shotgun metagenomics, we observe that the microbiome structure based on resistome and microbial taxonomy indicate that groups and time point accounted for $63 \%$ and $60 \%$ of the variation respectively (Fig S2a, i and iii). This observation highlights a direct relationship between microbiota and its resistome. Indeed, the clusters observed here also map to groups at specific time points. To unravel this relationship, 2,674 genomes were assembled from 24 metagenomes, and after quality control, 638 genomes (Fig. 5) were retained. Of these, 599 (93.9\%) were taxonomically classified as bacteria and $10(1.6 \%)$ as archaea at kingdom level. Of the bacteria, 29 (4.6\%) were unclassified and therefore represented potentially unculturable and /or novel prokaryotic taxa. At the lower taxonomic ranks i.e. family and genus levels, there represented 163 (25.6\%) and 231 (36.21\%) of the microbiota respectively. In the phylogenetic tree (Fig. 5), most of these unclassified phyla clustered with Bacteriodetes, Firmicutes and Lentisphaerae. Similarly, Bacteriodetes, Firmicutes and Proteobacteria accounted for $75.4 \%$ of observed taxa. We observed that AMR genes were generally carried by a wide range of bacteria across phyla but a small cluster carried genes across class of antibiotics. This cluster was exclusively observed in piglets at T2 and T3, it mostly contained phylum Proteobacteria and dominated by Escherichia coli and Clostridium species

\section{Discussion}

In this study, we assess the relationship between the resistome and microbiota, exploiting the resolution provided by shotgun sequence data. Here, we compare the granularity of two data sets - a) contigs and genomes assembled from shotgun metagenome sequencing data and b) OTUs from $16 \mathrm{~S}$ rRNA gene sequence data as well as gene copy count using qPCR. We target four critical time points on a swine production farm, i.e. farrowing, weaning, growing and finishing, where batch antibiotic treatment (metaphylaxis) was administered. The analysis was motivated by our previous work [11], which concluded that AMR gene levels on this farm were in a state of equilibrium, or saturated, but the antibiotics remained relatively effective in reducing the frequency of infections and mortality. We therefore sought to unravel the microbiome characteristics that underwrite such a 
phenomenon using amplicon and shotgun sequence data. In doing so, we generate critical insights needed to design strategies that limit welfare impact of disease whilst controlling the spread of antimicrobial resistance on farms.

\section{Characteristics of the gut resistome}

The sow microbiome exhibited limited variation in abundance and diversity of antibiotic resistance genes, therefore most of the variation was attributed to piglets [35]. Partitioning this variance suggests that up to $66 \%$ may be due to chlorotetracycline and tylosin usage in these piglets. Resistome variability in piglets has previously been reported [35] and its reduction as piglets mature is in part due to natural microbial succession [1, 35]. In this study, we were unable to fully delineate between changes due to the latter and antibiotic usage. Tylosin usage in piglets is known to accelerate microbial succession [35]. However, unravelling this specific aspect was beyond the scope of this study. Fundamentally, we show the direct relationship between microbiota and its resistome, furthermore, the characteristics of the resistome, mode of action for resistance and taxonomic profiles have led us to hypothesise that the marked changes seen at T3 in both piglets and sows may have different precursors.

\section{Changes due to chlorotetracyline and tylosin use}

Antibiotic-driven changes of the pig gut microbiome take up 14 days to manifest and may last for months [36, 37]. Here, the piglets had been administered chlortetracycline and acidified water at T2, but the dramatic changes in the selected amplicon resistome, i.e. abundance were observed 28 days later at T3. This may indicate a delayed response attributable to acidified water. At T3, post chlortetracycline administration the full resistome was characterised by an increase in diversity and abundance of tetracycline resistance encoding genes $(P>0.01)$, for example, tet44, tetW and tetO. Indeed this is reflected as an upregulation of tetracycline ribosomal protection protein was observed, which works by protecting the bacterial ribosome from binding the antibiotic tetracycline [38]. All of this points towards tetracycline-driven changes as reported elsewhere [39].

Beyond this, there was a down regulation of the MDR regulator mechanism which modulates resistance to aminoglycosides and aminocumarins, suggesting a counter selection. This would be expected to impede the functionality of efflux pump mechanisms which confer multi-drug resistance via the bacterial two-component signal transduction system $C p x A R[40,41]$. Such an effect would likely compromise the survival of certain microbial populations [42]. Finally, the resistome of piglets at T3 was also characterised by an increase in diversity of genes that encode resistance to glycopeptides and metronidazole, although this indicates co-selection, the mechanism behind this remains unclear. We had expected that the use of antibiotics would result in a reduction of both microbial and resistome diversity, however the use of chlortetracycline was associated with a significant increase in Shannon index [39], suggesting an increase in unique taxa and their abundance. Indeed, this increase is exhibited by a significant reduction of Verrumicrobiaceae and an increase of Bacteroidaceae. Low abundance of the former in piglets under similar conditions has been reported elsewhere [43].

Earlier studies have shown that sub therapeutic administration of tylosin in pigs resulted in a much larger effect than chlortetracycline [35, 43, 44]. In this study, therapeutic levels were used (265.8 mg/PCU tylosin, $103.2 \mathrm{mg} / \mathrm{PCU}$ chlortetracycline), and most of the variance in piglets was at T4 after tylosin administration. This could represent a combined effect of both antibiotics, however, if we assume that most of variation attributable to tetracycline was manifested at T3, then tylosin would account for a much larger effect [35, 44]. In this case, counter-selection i.e. chlortetracycline causing a suppression of efflux pump mechanism, may have resulted in microbial population that are more susceptible to antibiotics, leading to a much larger effect when tylosin was subsequently used. The response to this was an upregulation of efflux pumps specific for macrolides and this supports export of chemicals associated with macrolide activity out of the bacterial cell [45]. Interestingly, ermB and InuC remain abundantly stable across the three time points, which is possibly an indication of legacy use of this antibiotic on this farm. It is also possible that the full extent of tylosin's impact on this system was in the weeks beyond our selected time points.

The changes to microbiome structure and taxonomic distribution at T4 are comparable with that observed for the resistome, for example; although lower than T3 the Shannon index at T4 is significantly higher than in sows. It is likely that changes at T4 were more taxa-specific, which is why they account for significantly more variation in the resistome [35,44]. To this effect, a reduction in the abundance of phyla Bacteriodetes, Actinobacteria and Proteobacteria but an increase in Firmicutes might explain the level of change.

\section{Changes due to a potentially undetected infection in sows}

The sows did not receive antibiotics during this experiment, however they exhibited marked microbiome structural, resistome and taxonomic changes indicating an undetected incursion. Certain characteristics point towards an infection. Firstly, despite comparable microbial diversity, resistome and taxonomic characteristics were markedly different between sows and piglets at T3 i.e. sows exhibited an up regulation in Class A \& B betalactamases which catalyse the hydrolysis of the beta lactam ring using serine and zinc-based enzymes respectively [46]. On the other hand, multidrug efflux pump which are usually down-regulated by upstream pump operon transcription [1, 47]. Such a change is likely to represent an increase in microbial multidrug resistance phenotype for beta-lactams among the sows but the opposite would be expected in piglets [48]. Furthermore, an over representation of genes that encode resistance specifically those that modulate fluoroquinolone resistance, is likely an indication of legacy of use on the farm. Finally, a gradual increase of genes in piglets suggests continuum of exposure via microbial succession hastened or otherwise by antibiotic use [35]. 
Secondly, viral sequences reveal a marked increase in the abundance of Siphoviridae, Phycodnaviridae, Podoviridae and Smascoviridae. Siphoviridae is associated with Bordetella bronchiseptica, the causative agent of atrophic rhinitis [49]. Indeed, we observe a marked increase (T3) of sequences of the family to which Bordetella species belong. (Fig S7). On the other hand, Phycodnaviridae primarily affects algae [50], suggesting a potential environmental incursion. Smascoviridae, specifically of genus Porprismacovirus has previously been reported in pigs, however its potential to cause disease remains unknown. It is noteworthy that Adenoviridae sequences were also detectable in sows at T3 and these are known to cause mild gastroenteritis [51]. Crucially, the significantly higher abundance of Verrucomicrobiaceae in sows supports the notion of an infection as members in this family i.e. Akkermansia are associated with attenuation of gut inflammatory response [43].

\section{Characterising AMR gene carrying taxa}

While it is widely accepted that the vast majority of AMR genes are carried by the uncultured fraction of a microbiome, here we have used 682 near complete genomes across major culturable taxa to show that majority of the AMR genes are carried by Firmicutes and Proteobacteria. This observation supports the notion of a direct relationship between microbiota and its resistome given that these phyla account for $58 \%$ of microbial population in this study. Furthermore, we show that a group of Proteobacteria i.e. Escherichia coli and Clostridium sp, were exclusively recovered from piglets and carried a variety of genes encoding resistance to classes of antibiotics i.e. the phenotype of MDR would be expected in this group of bacteria. This further underscores the impact of antibiotic use in this group.

\section{Relevance to swine production}

Unravelling gut microbiome characteristics is an essential step in developing strategies that maximise antibiotic utility, nutrient extraction [52], mucosal immune-modulation [52] to improve welfare outcomes [37] for sustainable livestock production systems. To do so, we ought to use methodology that accurately capture these characteristics. Here, we show some differences in the utility of 16S rRNA gene metabarcoding and shotgun metagenomic sequencing and conclude that; a) the former could cost effectively be used to screening for patterns in longitudinal studies, b) and the latter for granular examination of what they represent.

While most studies have focused on bacterial shotgun sequences, here we show that viral sequences can be used to support hypothesis investigations. To this effect we used viral sequences to investigate a suspected infection among sows, and the findings suggest that changes due to infections or otherwise could induce far larger microbiome changes than antibiotic use. It is therefore essential to account for such factors when analysing microbiome data.

Our original hypothesis here was that antibiotic use would restrict microbial diversity due to its bactericidal effect and this would result in an overrepresentation of genes encoding the corresponding resistance. The findings show that antibiotic use in pigs is associated with changes in the microbial structure, abundance, and typically an increase in diversity. The changes in the resistome can take between 14-28 days to manifest and depending on the method of detection, remain detectable for a long time. All this highlights the integral relationship between microbial populations and the genes they carry, and the opportunities and challenges of exploiting them.

\section{Conclusions}

Gut microbiota and its resistome changed in response to antibiotic usage and these changes remain detectable for a long time. However, we ought to be prudent as similar change can be elicited by other factors, here we show that these are delineable using shotgun sequences including but not limited to the bacterial kingdom. We therefore recommend using 16S rRNA amplicon to screen for signatures and shotgun sequence data for their granular analysis.

\section{Abbreviations}

AMR: Antimicrobial resistance

16S rRNA: 16 Sub-unit of Ribosomal Ribonucleic acid

MDR: Multi-drug resistance (in reference to phenotype)

DNA: Deoxyribonucleic acid

QIIME-2: Quantitative Insights into Microbial Ecology-2 ${ }^{\mathrm{TM}}$

OTU: Operational taxonomic unit

qPCR: quantitative polymerase chain reaction

UCl: Unique chromosomal identifier

AC: antibiotic class 
T: Time

SI: Sample identification

SD: Standard deviation

MLS: Macrolide Lincosamides and Streptogramins

CCR: cross class resistance (in reference to genotype)

ABC efflux pumps: ATP binding cassette efflux pumps

mg/PCU: milligram/per population correction unit

\section{Declarations}

\section{Ethics approval}

In this study we collected samples considered environmental i.e. faecal droppings, therefore it was not mandatory to obtain ethical approval. Nonethe-less we our experimental protocol was approved by the Easter bush veterinary ethics review committee VERC.

\section{Consent for publication}

All co-authors on this manuscript consented to the publication of this work

\section{Data availability}

The raw sequences from the shotgun sequencing for this study are publicly available on EMBL-EBI under accession number PRJEB34736. The amplicon sequence data sets are publicly available on the NCBI Sequence Read Archive (16S rRNA gene metabarcoding data - BioProject accession number PRJNA557844)

\section{Funding}

$\mathrm{AM}, \mathrm{SB}, \mathrm{MB}$, and DG are supported by the Biotechnology and Biological Sciences Research Council (BBSRC) through the Roslin Institute Strategic Programme "Control of Infectious Diseases" (BBS/E/D/20002173 and BBS/E/D/20002174). AM was specifically supported by BBSRC Future Leader Fellowship (BB/P007767/1) and University of Edinburgh's ISSF3 (1S3-RI.0919/20). The project was funded by the UK Research and Innovation (UKRI) AMR Cross Council Initiative, administered by the Natural Environment Research Council (NERC reference: NE/N020162/1) (Principal Investigator: Dr Alexander Corbishley). MH's time is support by the Scottish Government.

\section{Acknowledgment}

Special thanks to Dr. Laura Glendinning for pre-submission internal review of this manuscript

\section{Authors information}

\section{Affiliations}

The Roslin Institute and Royal (Dick) School of Veterinary Studies, University of Edinburgh, Midlothian, Edinburgh, United Kingdom

Adrian Muwonge, Shih Barbara, Mark Bronsvoort, David L. Gally and Alexander Corbishley

Animal and Veterinary Sciences, Scotland's Rural College (SRUC), Edinburgh, United Kingdom

Jolinda Pollock

SRUC Veterinary Services, Scotland's Rural College (SRUC), Edinburgh, United Kingdom

Jolinda Pollock \& Michael R. Hutchings

Department of Veterinary Services, Ministry of Fisheries and Livestock, Lusaka, Zambia

Geoffrey Mainda

\section{Contribution}


AM \& BS analytical design and analysis, manuscript writing, DJP \& GM Field and laboratory experiment, AC, DG, MB and MH experimental design and funding acquisition

\section{Corresponding author}

Adrian Muwonge, Adrian.muwonge@roslin.ed.ac.uk

\section{References}

1. Looft T, Johnson TA, Allen HK, Bayles DO, Alt DP, Stedtfeld RD, et al. In-feed antibiotic effects on the swine intestinal microbiome. ProcNatlAcadSciUSA. 2012;109:1691-6.

2. Manyi-Loh C, Mamphweli S, Meyer E, Okoh A. Antibiotic Use in Agriculture and Its Consequential Resistance in Environmental Sources: Potential Public Health Implications. Molecules [Internet]. Multidisciplinary Digital Publishing Institute; 2018 [cited 2021 Jan 26];23:795. Available from: http://www.mdpi.com/1420-3049/23/4/795.

3. Castanon JIR. History of the use of antibiotic as growth promoters in European poultry feeds. Poult. Sci. 2007.

4. Van Boeckel TP, Gandra S, Ashok A, Caudron Q, Grenfell BT, Levin SA, et al. Global antibiotic consumption 2000 to 2010 : An analysis of national pharmaceutical sales data. Lancet Infect Dis. 2014.

5. FAO. Antimicrobial R; Animal production [Internet]. FAO. 2020 [cited 2021 Jan 26]. p. 2. Available from: http://www.fao.org/antimicrobialresistance/key-sectors/animal-production/en/.

6. Zeineldin M, Aldridge B, Lowe J. Antimicrobial effects on swine gastrointestinal microbiota and their accompanying antibiotic resistome. Front. Microbiol. 2019.

7. Veterinary Medicines Directorate. UK One Health Report - Joint report on antibiotic use and antibiotic resistance, 2013-2017. 2019;2013-7. Available from:

https://assets.publishing.service.gov.uk/government/uploads/system/uploads/attachment_data/file/775075/One_Health_Report_2019_v45.pdf.

8. Xia Y, Li B, Yang Y, Li L, Tiedje JM, Zhang T. Metagenomic Assembly Reveals Hosts of Antibiotic Resistance Genes and the Shared Resistome in Pig, Chicken, and Human Feces. Environ Sci Technol. 2016;50:420-7.

9. De R. Metagenomics. Aid to combat antimicrobial resistance in diarrhea. Gut Pathog. 2019.

10. Segata N, Waldron L, Ballarini A, Narasimhan V, Jousson O, Huttenhower C. Metagenomic microbial community profiling using unique cladespecific marker genes. Nat Methods. 2012.

11. Pollock J, Muwonge A, Hutchings MR, Mainda G, Bronsvoort BM, Gally DL, et al. Resistance to change: AMR gene dynamics on a commercial pig farm with high antimicrobial usage. Sci Rep. 2020;10.

12. Comeau AM, Douglas GM, Langille MGI. Microbiome Helper: a Custom and Streamlined Workflow for Microbiome Research. mSystems. $2017 ; 2$.

13. Bolger AM, Lohse M, Usadel B. Trimmomatic: A flexible trimmer for Illumina sequence data. Bioinformatics. 2014;30.

14. Ginestet C. ggplot2: Elegant Graphics for Data Analysis. J R Stat Soc Ser A (Statistics Soc. 2011.

15. Li D, Liu CM, Luo R, Sadakane K, Lam TW. MEGAHIT: An ultra-fast single-node solution for large and complex metagenomics assembly via succinct de Bruijn graph. Bioinformatics. 2015;31.

16. Seemann T. ABRicate: mass screening of contigs for antimicrobial and virulence genes. 2018. p. 1.

17. Steven ML, Chris D, Noelle RN, Adam D, Spencer A, Ross D, et al. MEGARes: an antimicrobial resistance database for high throughput sequencing. Nucleic Acids Res. 2017;4:D574-80.

18. Von Meijenfeldt FAB, Arkhipova K, Cambuy DD, Coutinho FH, Dutilh BE. Robust taxonomic classification of uncharted microbial sequences and bins with CAT and BAT. Genome Biol. 2019;20.

19. Krawczyk PS, Lipinski L, Dziembowski A. PlasFlow: predicting plasmid sequences in metagenomic data using genome signatures. Nucleic Acids Res. 2018;46.

20. Schep N, K Kummerfeld A. S. iheatmapr: Interactive complex heatmaps in R. J Open Source Softw. 2017;2.

21. Love MI, Huber W, Anders S. Moderated estimation of fold change and dispersion for RNA-seq data with DESeq2. Genome Biol. $2014 ; 15$.

22. Lê Cao K. Statistical analysis of microbiome data with R. YinglinXia, JunSun, Ding-GenChen. (2018). Singapore: Springer. 505 pages, ISBN: $978-$ 981-13-1533-6. Biometrical J. 2019;61.

23. Bates D, Mächler M, Bolker BM, Walker SC. Fitting linear mixed-effects models using Ime4. J Stat Softw. 2015;67.

24. Esarey J, Sumner JL. Exploring interactions with continuous predictors in regression models. Comp Polit Stud. $2018 ; 51$.

25. Li H, Durbin R. Fast and accurate short read alignment with Burrows-Wheeler transform. Bioinformatics. 2009;25:1754-60.

26. Houtgast EJ, Sima VM, Bertels K, Al-Ars Z. Hardware acceleration of BWA-MEM genomic short read mapping for longer read lengths. Comput Biol Chem. 2018;75. 
27. Stewart RD, Auffret MD, Snelling TJ, Roehe R, Watson M. MAGpy: A reproducible pipeline for the downstream analysis of metagenomeAssembled genomes (MAGs). Bioinformatics. 2019;35.

28. Hyatt D, Chen GL, LoCascio PF, Land ML, Larimer FW, Hauser LJ. Prodigal: Prokaryotic gene recognition and translation initiation site identification. BMC Bioinformatics. 2010;11.

29. Carattoli A, Zankari E, Garcia-Fernandez A, Larsen M, Lund O, Villa L, et al. PlasmidFinder and pMLST: in silico detection and typing of plasmids. Antimicrob Agents Chemother. 2014.

30. Parks DH, Imelfort M, Skennerton CT, Hugenholtz P, Tyson GW. CheckM: Assessing the quality of microbial genomes recovered from isolates, single cells, and metagenomes. Genome Res. 2015;25.

31. Asnicar F, Thomas AM, Beghini F, Mengoni C, Manara S, Manghi P, et al. Precise phylogenetic analysis of microbial isolates and genomes from metagenomes using PhyloPhIAn 3.0. Nat Commun. 2020;11.

32. Bateman A, Martin MJ, Orchard S, Magrane M, Agivetova R, Ahmad S, et al. UniProt: The universal protein knowledgebase in 2021. Nucleic Acids Res. 2021;49.

33. Buchfink B, Xie C, Huson DH. Fast and sensitive protein alignment using DIAMOND. Nat. Methods. 2014.

34. Pollock J, Muwonge A, Hutchings MR, Mainda G, Bronsvoort BM, Gally DL, et al. Resistance to change: AMR gene dynamics on a commercial pig farm with high antimicrobial usage. Sci Rep. 2020;10.

35. Kim HB, Borewicz K, White BA, Singer RS, Sreevatsan S, Tu ZJ, et al. Microbial shifts in the swine distal gut in response to the treatment with antimicrobial growth promoter, tylosin. Proc Natl Acad Sci U S A. 2012;109.

36. Schokker D, Zhang J, Zhang LL, Vastenhouw SA, Heilig HGHJ, Smidt H, et al. Early-life environmental variation affects intestinal microbiota and immune development in new-born piglets. PLoS One. 2014;9.

37. Guevarra RB, Lee JH, Lee SH, Seok MJ, Kim DW, Kang BN, et al. Piglet gut microbial shifts early in life: Causes and effects. J. Anim. Sci. Biotechnol. 2019.

38. Li W, Atkinson GC, Thakor NS, Allas U, Lu CC, Yan Chan K, et al. Mechanism of tetracycline resistance by ribosomal protection protein Tet(0). Nat Commun. 2013;4.

39. Ghanbari M, Klose V, Crispie F, Cotter PD. The dynamics of the antibiotic resistome in the feces of freshly weaned pigs following therapeutic administration of oxytetracycline. Sci Rep. 2019;9.

40. Hirakawa H, Nishino K, Hirata T, Yamaguchi A. Comprehensive studies of drug resistance mediated by overexpression of response regulators of two-component signal transduction systems in Escherichia coli. J Bacteriol. 2003;185.

41. Kurabayashi K, Hirakawa Y, Tanimoto K, Tomita H, Hirakawa H. Role of the CpxAR two-component signal transduction system in control of fosfomycin resistance and carbon substrate uptake. J Bacteriol. 2014;196.

42. Benomar S, Evans KC, Unckless RL, Chandler JR. Efflux pumps in Chromobacterium species increase antibiotic resistance and promote survival in a coculture competition model. Appl Environ Microbiol. 2019;85.

43. Song D, Peng Q, Chen Y, Zhou X, Zhang F, Li A, et al. Altered Gut Microbiota Profiles in Sows and Neonatal Piglets Associated with Porcine Epidemic Diarrhea Virus Infection. Sci Rep. 2017;7.

44. Holman DB, Chénier MR. Temporal changes and the effect of subtherapeutic concentrations of antibiotics in the gut microbiota of swine. FEMS Microbiol Ecol. 2014;90.

45. Pyörälä S, Baptiste KE, Catry B, van Duijkeren E, Greko C, Moreno MA, et al. Macrolides and lincosamides in cattle and pigs: Use and development of antimicrobial resistance. Vet. J. 2014.

46. Connelly S, Fanelli B, Hasan NA, Colwell RR, Kaleko M. Oral metallo-beta-lactamase protects the gut microbiome from carbapenem-mediated damage and reduces propagation of antibiotic resistance in pigs. Front Microbiol. 2019;10.

47. Hansen LH, Sørensen SJ, Jørgensen HS, Jensen LB. The prevalence of the OqxAB multidrug efflux pump amongst olaquindox-resistant Escherichia coli in pigs. Microb Drug Resist. 2005;11.

48. Blanco P, Hernando-Amado S, Reales-Calderon J, Corona F, Lira F, Alcalde-Rico M, et al. Bacterial Multidrug Efflux Pumps: Much More Than Antibiotic Resistance Determinants. Microorganisms. 2016;4.

49. Petrovic A, Kostanjsek R, Rakhely G, Knezevic P. The First Siphoviridae Family Bacteriophages Infecting Bordetella bronchiseptica Isolated from Environment. Microb Ecol. 2017;73.

50. Wilson WH, Van Etten JL, Allen MJ. The Phycodnaviridae: The story of how tiny giants rule the world. Curr. Top. Microbiol. Immunol. 2009.

51. Sanford SE, Hoover DM. Enteric adenovirus infection in pigs. Can J Comp Med. 1983;47.

52. Wang WL, Xu SY, Ren ZG, Tao L, Jiang JW, Zheng SS. Application of metagenomics in the human gut microbiome. World J Gastroenterol. 2015;21.

\section{Figures}




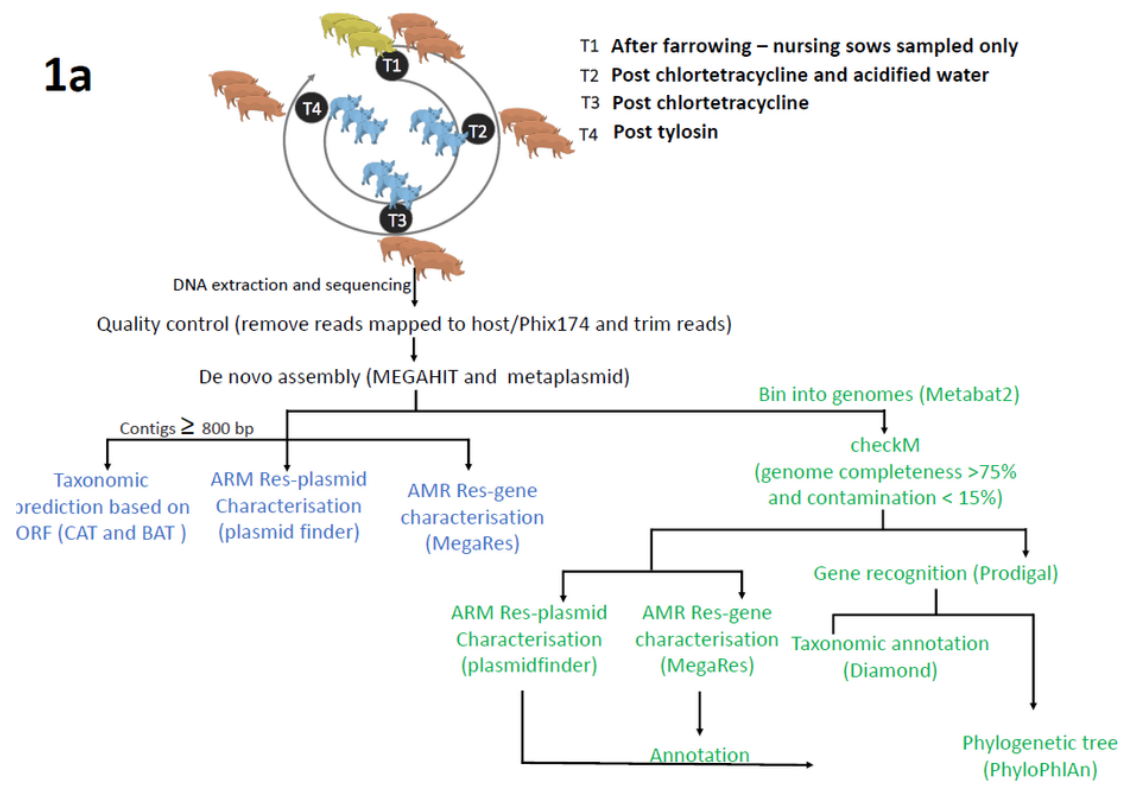

\section{1b}

Production cycle

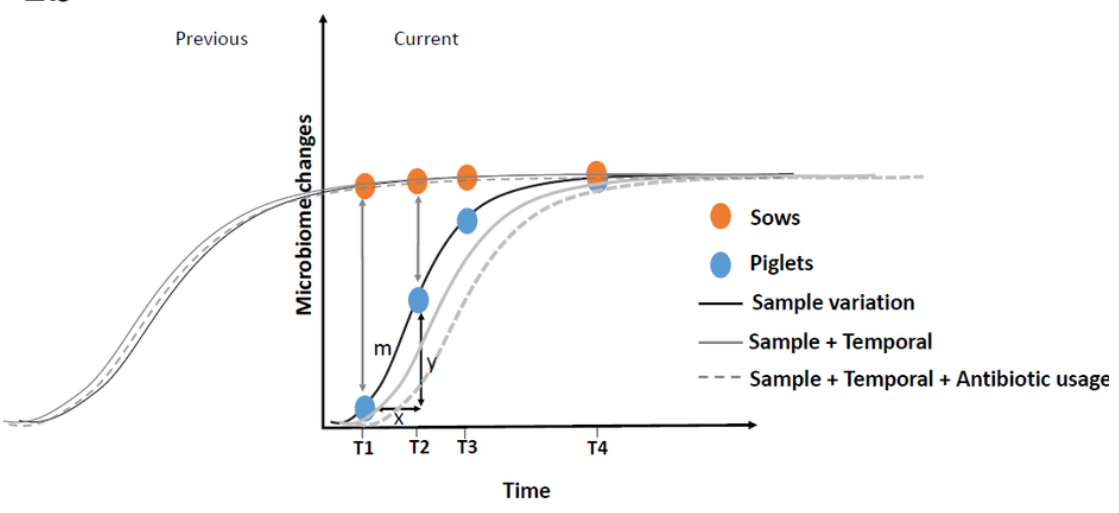

Figure 1

a. Shows the study design, two groups i.e. piglets and sows longitudinally sampled at T1, T2, T3 and T4. After DNA extraction, sequencing and quality control, the reads are assembled to feed two analytical approaches. In green, the reads are assembled and filtered to retain $>=800 \mathrm{bp}$ contigs, which are then annotated with AMR genes as well as taxonomic classification. In Blue, the reads are assembled into near complete genomes $>75 \%$ and $A M R$ genes, plasmids as well as taxonomic classification done. Fig $1 \mathrm{~b}$. Shows the hypothesis and assumption on which the analysis is done. In this study we have measured time represent as categorical increment $(x)$, analysed the microbiome therefore changes between individuals over time (y) can be established and $\mathrm{m}$ is the rate of change in the microbiome characteristics. We assume that $\mathrm{m}$ reduces as piglets grow which is why sows in general will show limited variation (Fig 4b). We assume that total sample variation will follow the black line, antibiotic use and other routine activities can affect the $\mathrm{m}$ therefore shift the line to the grey and broken line positions, and thus accounting for the variation attributable to these factors. This assumption is validated in figure (Fig $4 b$ ) 

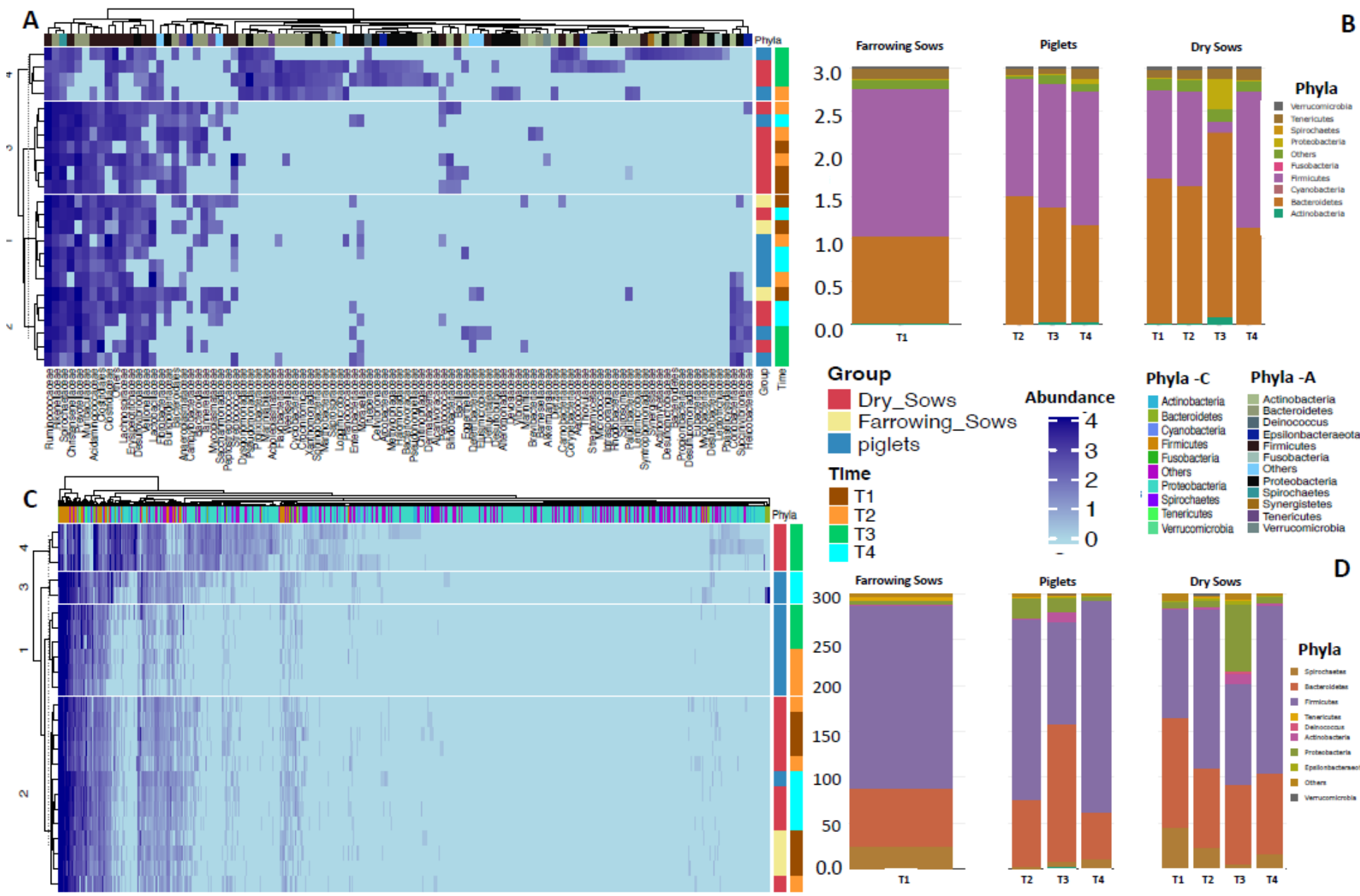

Family

Figure 2

Shows the taxonomic characteristic of the microbiome based on 16sRNA and shotgun metagenomic sequencing data. Panel A and C Shows the hierarchical clustering of family level abundance based on 16sRNA and shotgun sequencing data respectively. The latter's taxonomic assignment is based on contigs ( $>=800 \mathrm{bp}$ ). Panel B and D shows the taxonomic profile at phyla level base on 16sRNA and shotgun sequencing data for pig groups across the four time points. Primarily, this figure demonstrates the difference in granularity of the two sequencing methods and the strength of the taxonomic signal based on how well it clusters samples group and time. 


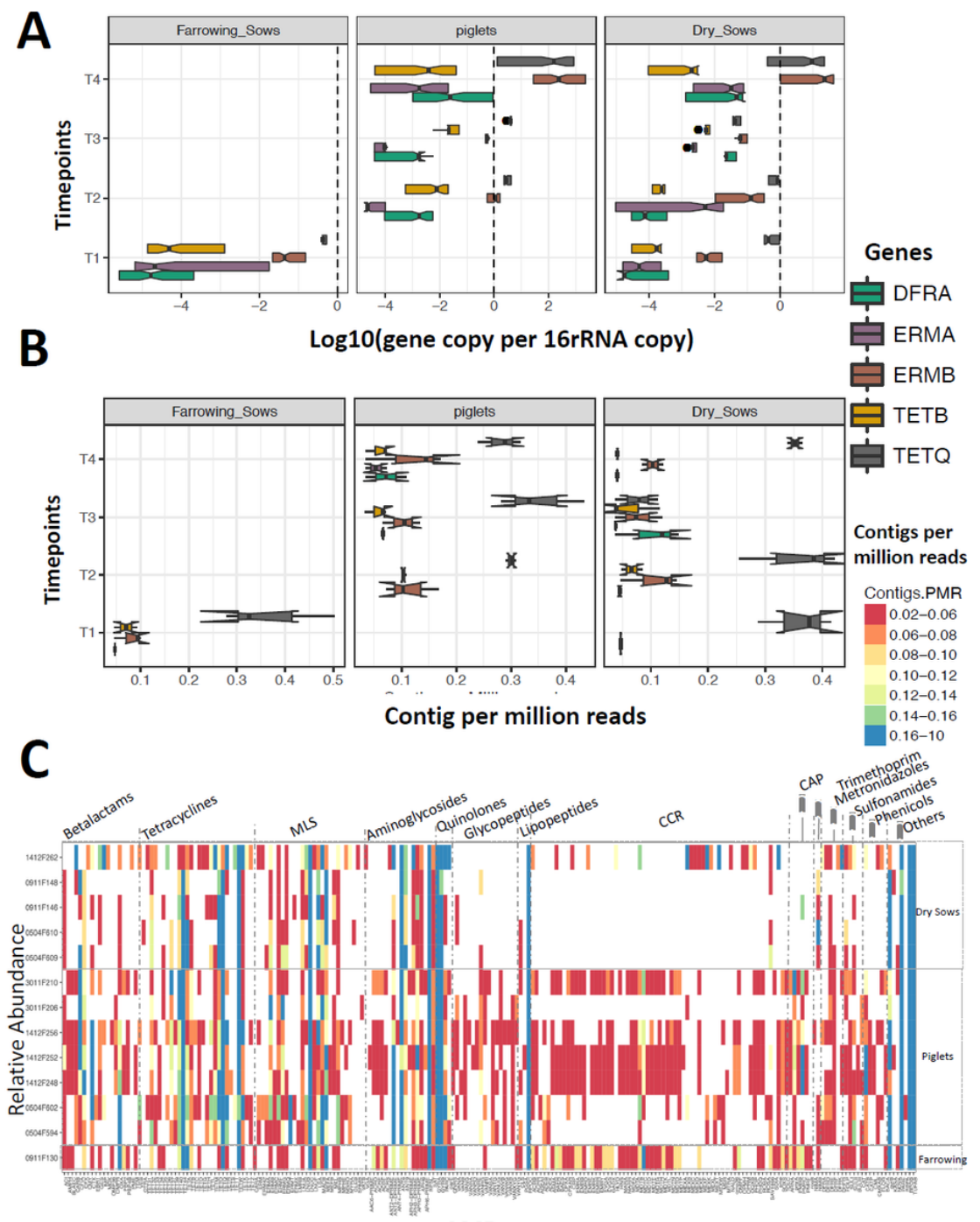

AMR-genes

Figure 3

Shows the resistome characteristics. Panel A shows the selected amplicon resistome, the copy-count of five genes normalised by 16 sRNA copy number. Panel $B$ is an analysis of the same genes based on shotgun data measures as contigs per million reads carrying the selected AMR genes) based on qPCR and shotgun sequencing data. These two panels provide a comparison in characteristics as detected using both sequence methods. Panel $\mathrm{C}$ shows the full resistome i.e. AMR genes clustered by antibiotic class. The fill colour shows the number of contigs per million reads. CCR cross class resistance, MLS- macrolides Lincomycines and streptogramins, CAP- Cationic antimicrobial peptides. Others = Elfamycin, Rifamycins 

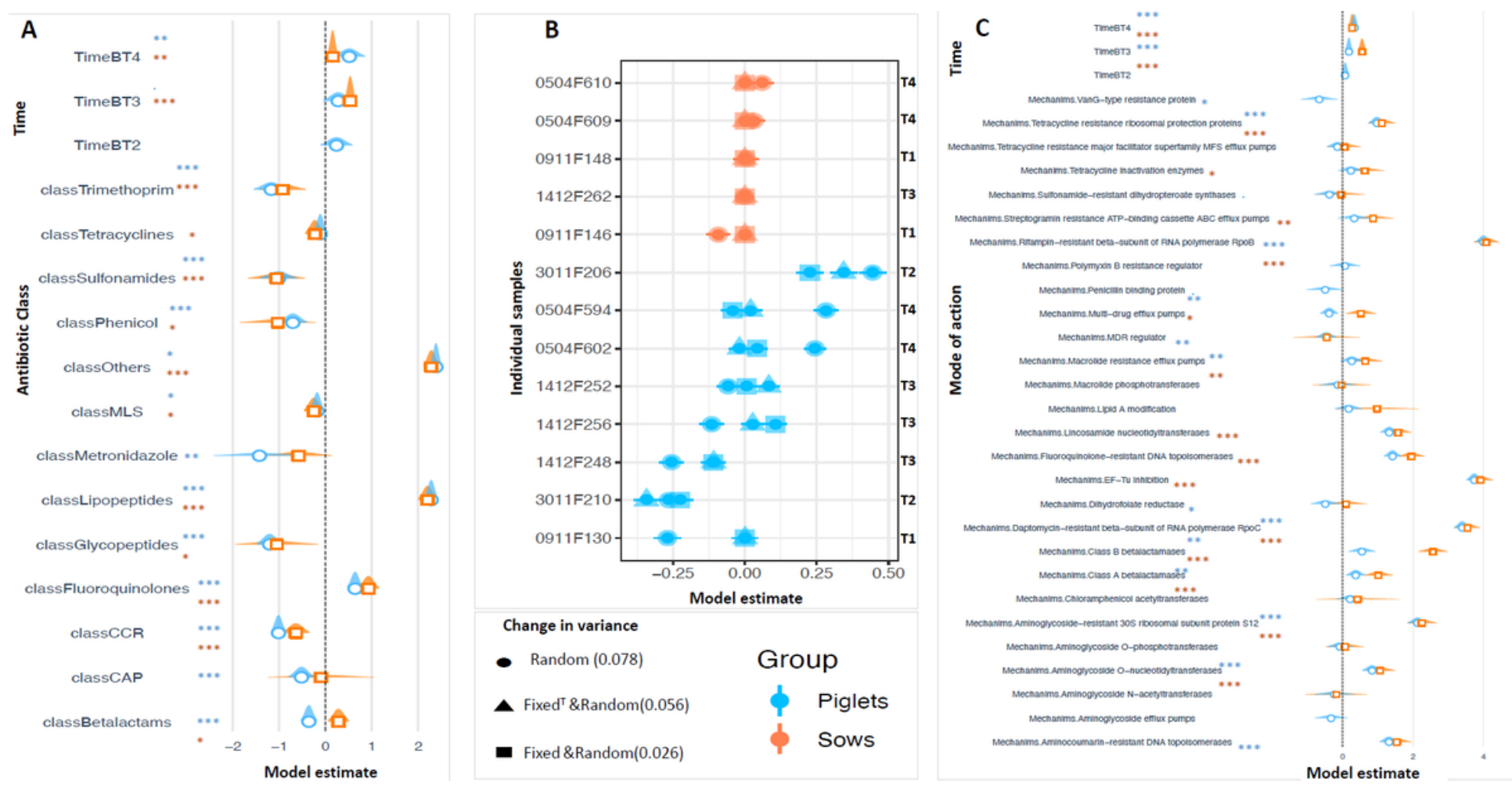

\section{Figure 4}

Shows the analysis aimed at partitioning attributable variation in gene count using a Poisson mixed effects model. We account for variation attributable to time and antibiotic use by sequentially adding the factors to the model calculating the difference in variance. Panel $A$, is the fixed model component showing changes in gene count attributable to antibiotic class, time in different pig groups. Panel B shows the random model component, here we show the change variance when the model only has the random component (Random), time is added as a fixed (FixedT \&Random) component and all fixed and random (Fixed \&Random) are added to the model. 


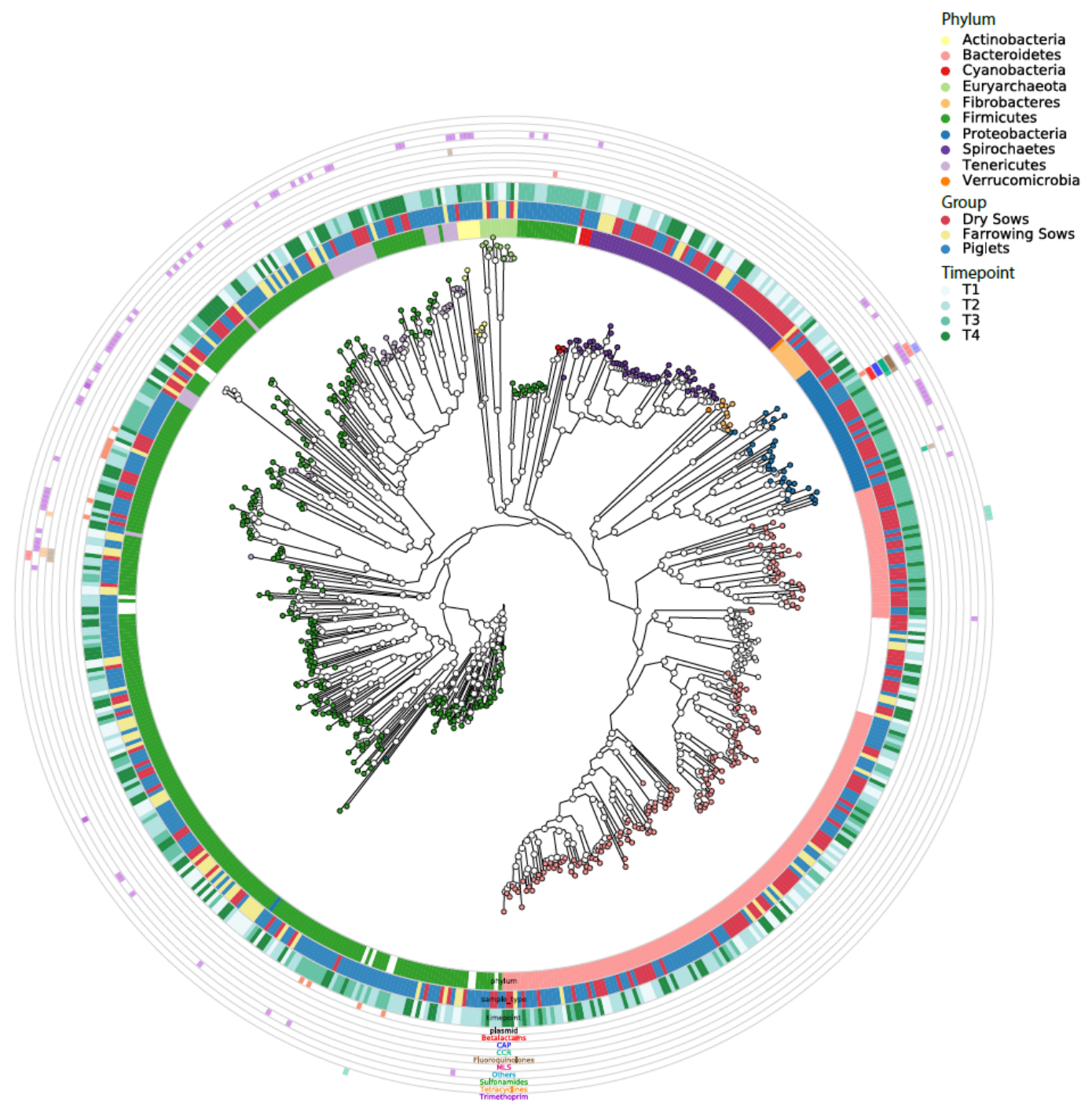

Figure 5

Shows the taxonomic units are carrying the AMR genes and plasmids. The taxonomic units are Metagenomics assembled genomes ( $\mathrm{n}=682$ ) with genome completeness $>75 \%$ and contamination $<15 \%$. Here the genomes are coloured by phyla (inner large ring). The larger two rings from the inner ring show the group and Time points. The smaller rings centripetally show hits on plasmids and antibiotic classes per genome colour intensity corresponds to abundance.

\section{Supplementary Files}

This is a list of supplementary files associated with this preprint. Click to download.

- FigS1.pdf

- FigS2.pdf

- FigS3.pdf

- Figs4.pdf

- FigS5.pdf

- FigS6.pdf

- Figs7.pdf 